\title{
Comparative analysis of antifriction polymers behaviour under deformation of spherical bearing
}

\author{
Anna Kamenskikh \\ Department of Computational Mathematics and Mechanics \\ Perm National Research Polytechnic University \\ Perm, Russia \\ anna_kamenskih@mail.ru
}

\author{
Maria Bartolomey \\ Department of Computational Mathematics and Mechanics \\ Perm National Research Polytechnic University \\ Perm, Russia \\ anna_kamenskih@mail.ru
}

\begin{abstract}
The stress-strain state of a spherical contact unit with a layer of antifriction polymer material is investigated within the framework of the problem of elastoplastic contact interaction with the account of friction on the contact surface. The statement of the problem of contact mechanical interaction of two elastic bodies through an elastoplastic layer of antifriction polymer material is made. The contact characteristics at different finite element meshes are investigated, optimal mesh parameters for numerical modeling are chosen and convergence of numerical solution of the problem is established by the example of axisymmetric problem. As a result, numerical studies have established the influence of the geometry of the structure and the material of the antifriction layer on the distribution of sites with different types of interaction in the contact area (adhesion, sliding and exfoliation), the value and character of the contact pressure and contact tangent stress. The analysis of the work of the three antifriction materials (fluoropolymer, antifriction composite material based on fluoropolymer with spherical bronze inclusions and molybdenum disulfide, modified fluoropolymer) for two variants of boundary conditions is made, the influence on the character of the contact interaction is established.
\end{abstract}

Keywords-contact interaction; polymer; friction; antifriction layer; finite element method

\section{INTRODUCTION}

The modern nanofilled and unfilled fluoropolymers, ultramolecular polyethylenes, nanomodified polymer materials and composites of various brands of domestic and foreign production find a wide application in the technical, medical, construction units working in the conditions of combined straining including conditions of contact interaction. At the same time the solution of the problem of design and units deformation with such polymers in the framework of solid mechanics assumes the existence of the base of physicomechanical, thermomechanical, friction and operating properties of the materials. In mechanical engineering, construction, medicine and other areas use of contact units with coverings and layers from polymer antifriction materials that work in the conditions of contact interaction is widespread. As a rule, it is expensive, difficult to repair responsible elements, which are subject to increased requirements for strength, reliability and durability. As examples of systems with contact layers and coverings, it is possible to note sliding bearings with a layer from composite material [1, 2], hip endoprosthesis with an antifriction layer [3], connection of a bone and joint cartilage [4], road covering [5], pistons [6], bearings of bridge spans [7] and so on. Often more than one contact surface, complex spatial geometry, complex materials in the mechanical behavior and conditions of loading are present in designs. At the same time to create favorable working conditions for contact units, a comparative analysis of the behavior of modern polymer antifriction materials in the framework of friction contact interaction on model samples and in real structures is an actual direction of the study. And large amount of research implies the construction of effective mathematical models describing the behavior of antifriction polymers and their numerical implementation.

Due to large number of studies of complex systems and structures with one or more sliding surfaces with antifriction coatings and layers, the contact problems of mechanics of multilayer medium and bodies with coatings and layers have been widely developed [8-10]. Significant number of works is devoted to the contact mechanical interaction of elastic layered medium [11-14]: the contact of a spherical indenter or a system of dies with elastic layered semi-space is considered, and also the contact that occurs with a pressure upon the layered body. In [10], contact problems of bodies with coatings and layers are investigated within the framework of the theory of elasticity and viscoelasticity. Approaches to the solution of elastoplastic contact problems are described in [15-19]. Taking into account the significant nonlinearity of contact problems, effective numerical methods for solving them are developed. Let us note the examples of using the boundary element method (BEM) [20, 21 et al.], finite element method (FEM) [19, 22-24 et al.] and combined BEM-FEM [25, 26 et al.]. Many implementations of contact interaction of FEM are realized with the help of applied software systems ANSYS, ANSYS LS-DYNA, ABAQUS [25, 27 et al.].

In the work the numerical study of the behavior of the three polymer materials of the antifriction layer in the design of the spherical bearing of the span structures of bridges in the framework of the mechanics of contact interaction with friction is considered. (fig. 1). The calculation scheme includes: the top steel plate with a spherical polished segment -1 , the lower steel plate with a spherical cut -2 and spherical 
polymer antifriction layer with the main sliding surface $A B C$ 3 that separates them.

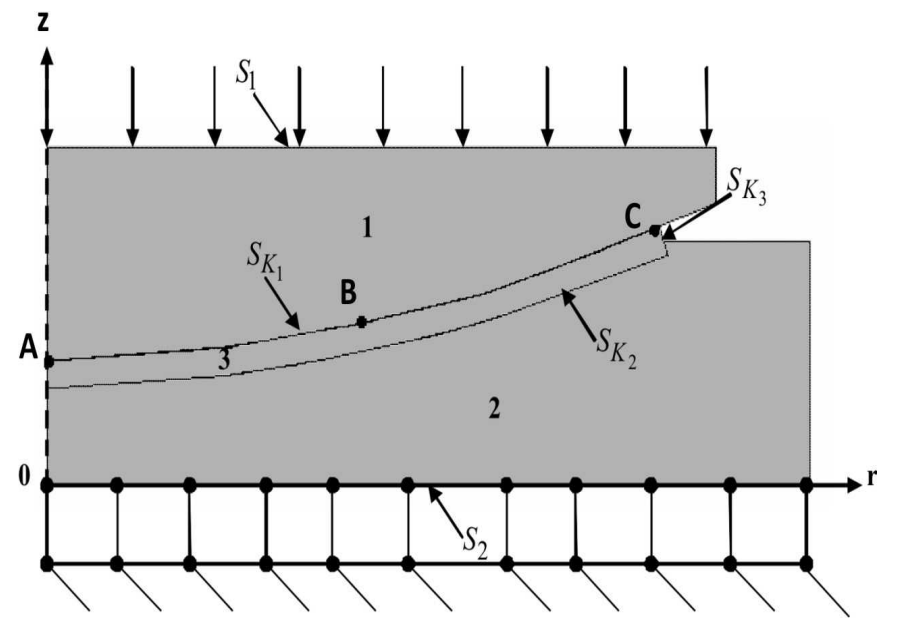

Fig. 1. Scheme of the spherical bearing.

The real design of the bearing with rated load of $2.5 \mathrm{MN}$ manufactured by Alfatech (Perm) on the project and with scientific and technical support of Ko Lumiere Ltd (Moscow) is modeled. At loading of the top plate there is a contact interaction between elastic steel plates 1,2 with an elastoplastic layer 3 from an antifriction polymer material. The construction of the bearing has the following characteristic dimensions: the maximum width of the structure is $b_{k}=0.274$ $\mathrm{m}$, the maximum height of the structure is $\mathrm{h}=4.83 \cdot 10^{-2} \mathrm{~m}$ and the thickness of the antifriction layer is $h_{p}=0.4 \cdot 10^{-3} \mathrm{~m}$.

Three materials of the antifriction layer are considered in the work: fluoropolymer (material 1), antifriction composite material based on fluoropolymer with spherical bronze inclusions and molybdenum disulfide (material 2) and modified fluoropolymer (material 3). Contact problems are implemented taking into account the friction, the friction coefficient is assumed constant and equal to 0.04. At Institute of Continuous Media Mechanics of the Ural Branch of Russian Academy of Science professor A.A. Adamov has executed a cycle of experimental studies of deformation characteristics of antifriction materials at difficult multistage stories of deformation with unloadings [28]. The cycle of experimental studies included: tests by determination of hardness of materials according to Brinell by indention of a ball with a $5 \mathrm{~mm}$ diameter; researches in the conditions of free compression, and also the constrained compression by pressing in special adaptation with a rigid steel holder of cylindrical samples with $20 \mathrm{~mm}$ diameter and length. All tests were carried out by the test car Zwick Z100SN5A. As a result, mechanical characteristics of the materials are received: elastic modulus $E$, Poisson's ratio $v$, deformation diagrams $\sigma_{z}-\varepsilon_{z}$ . Values of $E$ and $v$ for material 1 are equal to $5.45 \cdot 10^{8} \mathrm{~Pa}$ and 0.466 respectively; for material $2-8.6052 \cdot 10^{8} \mathrm{~Pa}$ and 0.4388 ; for material $3-8.638 \cdot 10^{8} \mathrm{~Pa}$ and 0.461 .

Two variants of calculation are considered for the analysis of the conditions of the bridge structure support on the top bearing plate: 1 . uniformly distributed pressure on the surface $S_{1} ; 2$. the equality of axial displacements by the coordinate $z$ on the surface $S_{1}$ is given additionally, and the surface $S_{1}$ remains plane during loading.

In the work, the contact problem of elastoplasticity is solved in the particular case of axisymmetric stress-strain state.

\section{DEFINING RELATIONS}

The general mathematical statement of elastic behavior of the material includes:

equilibrium relations

$$
\operatorname{div} \hat{\sigma}=0, \quad \vec{x} \in V ;
$$

geometrical relations

$$
\hat{\varepsilon}=\frac{1}{2}\left(\nabla \vec{u}+(\nabla \vec{u})^{T}\right), \quad \vec{x} \in V
$$

physical relations

$$
\sigma=\lambda I_{1}(\hat{\varepsilon}) \hat{I}+2 \mu \hat{\varepsilon}, \quad \vec{x} \in V_{1} \cup V_{2},
$$

where $\lambda$ and $\mu$ - Lame's parameters, $\hat{\sigma}-$ stress tensor, $\hat{\varepsilon}-$ strain tensor, $\vec{u}$ - vector of displacements, $\vec{x}$ - radius-vector of a random point, $I_{1}(\hat{\varepsilon})$ first invariant of strain tensor, $\hat{I}$ unit tensor, $V=V_{1} \cup V_{2} \cup V_{3}$ - design volume, $V_{1}$ - volume of the plate with a ball segment, $V_{2}$ - volume of the plate with a ball cutout, $V_{3}$ - volume of the polymeric layer.

To describe the behavior of the layer's material, the deformation theory of elastoplasticity is chosen, and physical relations are:

$$
\hat{\sigma}=\frac{2 \sigma_{u}}{3 \varepsilon_{u}}\left(\hat{\varepsilon}-I_{1}(\hat{\varepsilon}) \hat{I}\right)+3 K I_{1}(\hat{\varepsilon}) \hat{I}, \quad \vec{x} \in V_{3}
$$

where $\sigma_{u}=\sqrt{3 I_{2}(\hat{\sigma})}-$ intensity of stress tensor $\left(I_{2}(\hat{\sigma})-\right.$ second invariant of tensor $\hat{\sigma}), \varepsilon_{u}=\frac{2}{\sqrt{3}} \sqrt{I_{2}(\hat{\varepsilon})}-$ intensity of strain tensor $\left(I_{2}(\hat{\varepsilon})\right.$ - second invariant of tensor $\left.\hat{\varepsilon}\right), K-$ volume elastic modulus, $\sigma_{u}=\Phi\left(\varepsilon_{u}\right)$ - functional dependence determined by deformation diagram of the layer's material at uniaxial stress state.

The mathematical statement also includes kinematic boundary conditions on the surface $S_{2}$ :

$$
u_{z}=0, \vec{x} \in S_{2} \text {. }
$$

And static boundary conditions on the surface $S_{1}$ :

$$
\hat{\sigma} \cdot \vec{n}=\vec{P}, \vec{x} \in S_{1},
$$

the rest of the external surfaces are free: 


$$
\hat{\sigma} \cdot \vec{n}=0 .
$$

The system of equations (1) - (7) is complemented with boundary conditions on the contact surface $S_{K}=S_{K_{1}} \cup S_{K_{2}} \cup S_{K_{3}}$. The following types of contact interaction are considered:

- sliding with friction:

for static friction

$$
\sigma_{n}<0, \sigma_{n \alpha}<q \sigma_{n}, \vec{u}^{1}=\vec{u}^{2}
$$

for sliding friction

$$
\sigma_{n}<0, \sigma_{n \alpha}=q \sigma_{n}, u_{n}^{1}=u_{n}^{2}, u_{\tau}^{1} \neq u_{\tau}^{2}
$$

- exfoliation

$$
\left|u_{n}^{1}-u_{n}^{2}\right| \geq 0, \sigma_{n \alpha}=\sigma_{n}=0, \alpha=1,2 ;
$$

- full adhesion

$$
\vec{u}^{1}=\vec{u}^{2} .
$$

where $q$ - friction coefficient, $\alpha=1,2$ - indexes conditional numbers of two contacting surfaces, $u_{n}-$ displacements normal to the corresponding contact boundary, $u_{\tau}$ - displacements tangent to the corresponding contact boundary, $\sigma_{n}-$ stress normal to the contact boundary (三 contact pressure $P_{K_{1}}$ on the $\left.S_{K_{1}}\right), \sigma_{n \alpha}$ - stress tangent to the contact boundary ( $\equiv$ contact tangent pressure $\tau_{K_{1}}$ on the $S_{K_{1}}$ ).

In the second variant of the study, the system (1) - (11) is complemented with static constraints imposed on the surface $S_{1}$ :

$$
u_{z}(r, z)=U=\text { const }, \vec{x} \in S_{1} .
$$

The calculation variant 1 is realized in the frames of system of equations (1) - (11), variant 2 - system (1) - (12).

To study the mechanical behavior of the three materials of the antifriction layer in complex multi-stage deformation stories with unloading the typical series of experiments on free compression (at uniaxial stress state) in the compression strain range up to $10 \%$ and on the constrained compression (at uniaxial strain state) in the compression stress range up to 160 MPa were carried out [28].

Based on the analysis of the results of the experiments as a model for the behavior of the material the model of the deformation theory of elastoplasticity with elastic volumetric compressibility and nonlinear quasi-equilibrium diagram of deformation at free compression with minimum loading rate has been selected and identified.

To verify the availability of the selected behavior model of the material, a series of numerical experiments on solving boundary problems for tested samples using the ANSYS software package were performed. It is established that the numerical calculation with the use of the selected constitutive relations gives a good quantitative correspondence to the results of the experiment [29].

\section{CONSIDERATION OF THE RESULTS AND CONCLUSIONS}

The solution of axisymmetric contact problem is implemented. Finite element models using the deformation theory of elastoplasticity are constructed using the ANSYS software package. The axisymmetric 8th nodal element with approximation of the 2nd order and two unknown displacements in each node is used.

The finite element mesh is condensed in the layer, the size of the elements in the lower and top plates is increased gradient. The convergence of the solution from the number of elements $N$ in the thickness of the layer was investigated, four variants were considered: 2 and 4 elements, 8 and 16 elements. With reduce the size of an element by the layer thickness, the characteristic size of the layer elements decreases. In the lower and top plates the size of the elements increases in process of moving away from the contact surfaces When splitting a layer into 16 layers of elements, the total number of nodal unknowns is about 150 thousand, while there are about 1000 contact elements. The calculation takes about 2 minutes, the solution of the contact problem with application of automatic selection of the increment value algorithm for the external impact required 8 substeps of loading on 25 total iterations of performance of conditions on contact and friction. When using a mesh with 8 elements in the thickness of the layer, the total number of nodal unknowns is about 50 thousand, the contact elements - about 500, calculation time about 16 seconds; to solve the contact problem it is required according to 4 substeps on 12 iterations. All calculations were performed on the server platform Soc-1366 SuperMicro 4U 7046T-H6R (two six-core processors Soc-1366 Intel Xeon X5650).

The contact finite element pairs allowing to consider friction are applied on the surfaces of contact of the top and lower plates with a layer. Static and kinematic boundary conditions corresponding to the real nominal load during operation of the bearing part with a spherical segment in the span structures of bridges are imposed on the finite-element model: the distributed vertical load with level $P=54 \mathrm{MPa}$ is applied to the top plate.

The convergence of the solution of the contact problem on the contact parameters is considered: boundary of adhesionsliding areas, contact pressure and contact tangent stress. These values are of the greatest interest on the contact surface of the layer $S_{K_{1}}$ through which the spherical segment of the top plate is rotated. The influence of the number of elements $N$ in the thickness of the layer on the relative contact pressure $P_{K_{1}} / P$ and relative contact tangent stress $\tau_{K_{1}} / P$ (related to applied load) for variants 1 and 2 by the example of material 3 is shown in fig. 2. The convergence of the solution is also considered in [16]. 

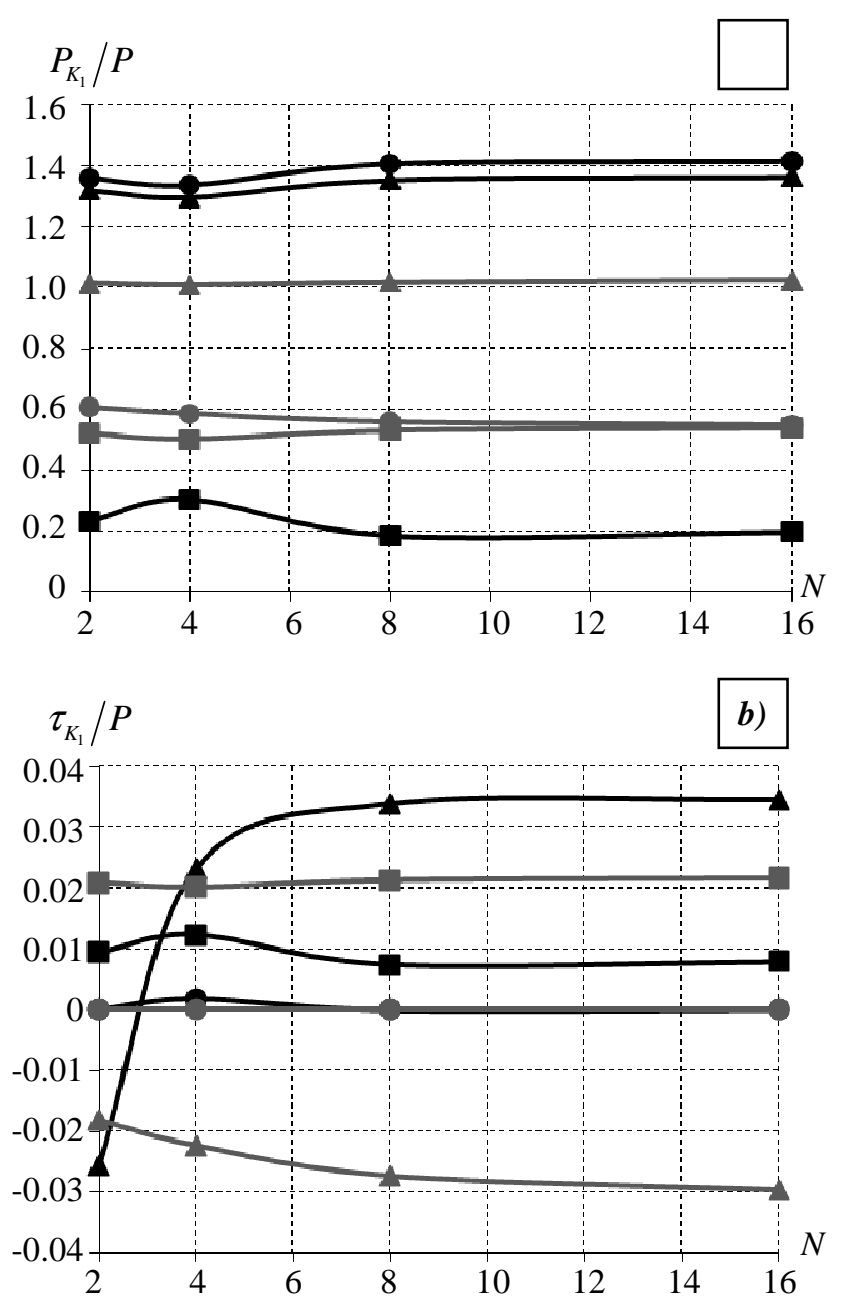

Fig. 2. The relative contact pressure (a) and tangent stress (b) in points A, B, $\mathrm{C}$ : grey line - variant 1 ; black line - variant 2; markers correspond to: circle point $\mathrm{A}$; triangle - point $\mathrm{B}$, square- point $\mathrm{C}$

Results presented in fig. 2 show their numerical convergence for both variants of calculations; therefore it is possible to be limited to number of layers $N$ of finite elements equal 8 or 16.

Further the distribution of relative contact pressure $P_{K_{1}} / P$ and relative tangent stress $\tau_{K_{1}} / P$ for the three studied materials in variants 1 and 2 at $N=16$ is considered (fig. 3 ).
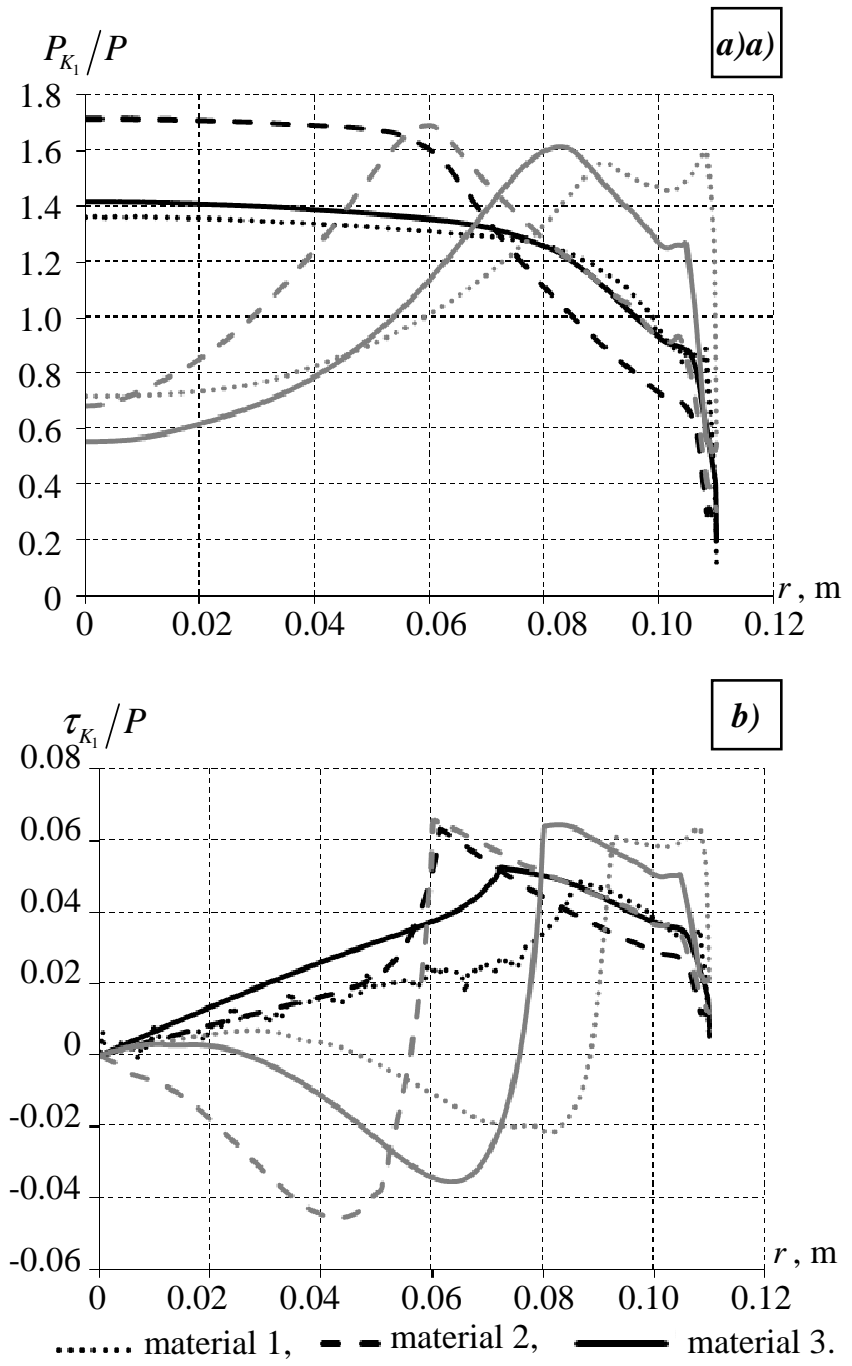

Fig. 3. The relative contact pressure (a) and tangent stress (b) on the surface $S_{K_{1}}$.

The ratio of values $P_{K_{1}} / P$ and $\tau_{K_{1}} / P$ and deformation fields obtained in all variants of calculations indicate the closeness of the stress-strain state of the polymer layer in the cohesion area to the state of the constrained compression, implemented during the testing of the materials [14].

For the bearing of variant 1 with all considered materials of the polymer layer, highly heterogeneous distribution of the relative contact pressure $P_{K_{1}} / P$ in the middle part of the spherical segment is observed: minimum in the center with a maximum in the area of transition of contact states (change of adhesion area with sliding area), closer to the edge of the layer contact pressure reduces due to the development of shear degrees of freedom during deformation of the polymer.

The relative tangent stresses $\tau_{K_{1}} / P$ in this variant of the boundary conditions on the surface $S_{1}$ also have evident minimum and maximum with the sign change in the neighborhood of the adhesion-sliding boundary. 
The reason for such heterogeneous distribution of relative contact pressures and tangent stresses in this variant of the boundary conditions on the surface $S_{1}$ of a relatively thin top steel plate is its axisymmetric bending, the surface $S_{1}$ becomes crowned upwards.

For variant 2 the relative contact pressure $P_{K_{1}} / P$ has a weakly expressed maximum in the middle part of the spherical segment and for materials 1, 3 its value is about $10 \%$ lower than the corresponding maximum of variant 1 . Relative tangent stresses $\tau_{K_{1}} / P$ grow uniformly to the boundary of the transition of the contact states, and then begin to decrease to zero at the free edge.

The adhesion-sliding boundaries of the top plate relative to the polymer layer for variants 1 and 2 are also different, and for each calculation variant their dependence (also true for $P_{K_{1}} / P$ and $\tau_{K_{1}} / P$ ) on the properties of the material of the antifriction layer at the same set of friction coefficient is observed.

Thus, the stress-strain state of the "plate-layer-plate" unit is analyzed within the framework of the contact interaction mechanics based on the numerical solution of the axisymmetric contact problem of elastoplasticity, the numerical assessment of the convergence of the obtained results is performed.

Boundaries of adhesion-sliding areas are established, the values and distribution of the contact pressure and contact tangent stress on the contact surfaces are determined, the influence of the properties of the three materials and the constructive restriction on the supporting surface of the top plate on the obtained numerical results is considered.

\section{CONCLUSION}

According to the results of the study as a model that more accurately reflects the work of the construction of the "platelayer-plate" unit the variant 2 with more rigid top plate to implement the conditions of support on the top plate of the bearing is recommended.

\section{Acknowledgment}

The reported study was funded by the RFBR according to research project No. 18-08-00903 a.

\section{References}

[1] W.A. Rakowski, S. Zimowski "Polyesterimide composites as a sensor material for sliding bearings", Composites: Part B engineering, vol. 37, pp. 81-88, 2006.

[2] M.V. Zernin, A.V. Mishin, N.N. Rybkin, S.V. Shil'ko "Hydrodynamic analysis of friction bearings, Part 1: Consideration of noncylindrical working surfaces", Journal of Friction and Wear, vol. 35, No. 5, pp. 396406, 2014/

[3] L.S. Pnchuk, V.I. Nikolaev, E.A. Tsvetkova, V.A. Goldade, Tribology and biophysics of artificial joints, 2006.

[4] I. Argatov "A general solution of the axisymmetric contact problem for biphasic cartilage layers", Mechanics Research Communications, No. 38 , pp. 29-33, 2011.
[5] Zh.B. Tukashev, L.A. Adilkhanova "Investigation of the stress-strain state of the pavement", Geology, geography and global energy, No. 2, pp. 163-166, 2010.

[6] O.V. Veretelnik, N.A. Tkachuk, S.Yu. Belik "Contact interaction of the piston with galvanic-plasma treatment of the lateral surface with the walls of the cylinder of the internal combustion engine", Bulletin of the National Technical University "KhPI". Computer Science and CAD, No. 22, pp. 32-39, 2012.

[7] G.I. Bogdanov, S.S. Tkachenko, S.A. Shulman, Bearings of bridges. Part 1: Textbook. allowance for university students, 2006.

[8] I.G. Goryacheva, Mechanics of Frictional Interaction, 2001.

[9] V.S. Nikishin, G.S. Shapiro, Spatial problems of the theory of elasticity for multilayer media, 1970.

[10] V.M. Alexandrov, S.M. Mkhitaryan, Contact problems for bodies with thin coatings and interlayers, 1983.

[11] N.A. Voronin "Calculation of the parameters of the elastic contact and effective characteristics of a topocomposite for the case of its contact with a spherical indentor", Journal of Friction and Wear, vol. 23, No. 6. pp. 583-596, 2002.

[12] S.J. Chidlow, M. Teodorescu, N.D. Vaughan "Predicting the deflection and sub-surface stress field within two-dimensional inhomogeneously elastic bonded layered solids under pressure", International Journal of Solids and Structures, vol. 48, No. 22-23, pp. 3243-3256, 2011.

[13] A.O. Sergici, G.G. Adams, S. Müftü "Adhesion in the contact of a spherical indenter with a layered elastic half-space", Journal of the Mechanics and Physics of Solids, vol. 54, No. 9, pp. 1843-1861, 2006.

[14] I.G. Goryacheva, E.V. Torskaya "Modeling of fatigue wear of a twolayered elastic half-space in contact with periodic system of indenters", Wear, vol. 268, No. 11-12, pp. 1417-1422, 2010.

[15] N.A. Voronin "Peculiarities and applied aspect of the mechanics of the contact interaction of a rigid spherical stamp with a vaporous plastic layered half-space", Mechanics and physics of processes on the surface and in the contact of solid bodies and machine parts, 2006.

[16] M.S. Drozd, M.M. Matlin, Yu.I. Sidyakin, Engineering calculations of elastoplastic contact deformation, 1986.

[17] V.M. Alexandrov, I.G. Kadomtsev, L.B. Tsaryuk "Axisymmetric contact problems for elastoplastic bodies", Journal of Friction and Wear, vol. 5, No. 1, pp. 16-26, 1984.

[18] W.Peng, B. Bhushan "Three-dimensional contact analysis of layered elastic/plastic solids with rough surfaces", Wear, vol. 249, No. 9, pp. 741-760, 2001.

[19] A. Rogovoy, B. Ivanov "Displacement formulation of the friction conditions on the contact surface", Computers and Structures, vol. 62, No. 1, hh. 133-139, 1997.

[20] C. Méchain-Renaud, A. Cimetiére, "BEM solution of two dimensional unilateral contact problems with friction by a new approach", Engineering Analysis with Boundary Elements, vol. 27, No. 3, pp. 269277, 2003.

[21] L. Rodríguez-Tembleque, F.C. Buroni, R. Abascal, A. Sáez "3D frictional contact of anisotropic solids using BEM", European Journal of Mechanics - A/Solids, vol. 30, No. 2, pp. 95-104, 2011.

[22] A.R. Khoei, M. Nikbakht "An enriched finite element algorithm for numerical computation of contact friction problems", International Journal of Mechanical Sciences, vol. 49, pp. 183-199, 2007.

[23] Y. Li, G.R. Liu, G.Y. Zhang "An adaptive NS/ES-FEM approach for 2D contact problems using triangular elements", Finite Elements in Analysis and Design, vol. 47, No. 3, pp. 256-275, 2011.

[24] N.G. Burago, A.B. Zhuravlev, I.S. Nikitin "Analysis of stress state of gte contact system disk-blade", Computational continuum mechanics, vol. 4 No. 2, pp. 5-16, 2011.

[25] J.A. González, K.C. Park, C.A. Felippa, R. Abascal "A formulation based on localized Lagrange multipliers for BEM-FEM coupling in contact problems", Computer Methods in Applied Mechanics and Engineering, vol. 197, No. 6-8, pp. 623-640, 2008.

[26] L. Rodríguez-Tembleque, R. Abascal "A FEM-BEM fast methodology for 3D frictional contact problems", Computers and Structures, vol. 88, No. 15-16. pp. 924-937, 2010. 
[27] C. Renaud, Z.-Q. Feng "BEM and FEM analysis of Signorini contact problems with friction", Computational Mechanics, vol. 31, pp. 390399, 2003.

[28] A.A. Adamov "Experimental study of the mechanical behavior of fluoroplastic-based composites operating at high pressures in thin layers", Materials of the VII Russian scientific and technical conference Mechanics of microinhomogeneous materials and destruction, 2012.

[29] A.A. Kamenskih, N.A. Trufanov "Regularities interaction of elements contact spherical unit with the antifrictional polymeric interlayer", Friction and Wear, vol.36, No. 2, pp. 170-176, 2015. 